\title{
Música en tiempos de crisis: precariedades del trabajo artístico y resurgimiento del apoyo mutuo en Chile
}

\section{Mutual aid among musical workers in Chile in times of crisis}

\section{Eileen Karmy}

Universidad de Playa Ancha, Valparaíso, Chile eileen.karmy@upla.cl

https://orcid.org/0000-0003-4174-9694

\section{Estefanía Urqueta}

Pontificia Universidad Católica de Valparaíso, Valparaíso, Chile estefania.urquetalapucv.cl https://orcid.org/0000-0003-4174-9694

\section{Resumen}

La pandemia global del Covid-19 generó la suspensión de conciertos y otras actividades musicales presenciales, agudizando la precariedad e inestabilidad de las y los trabajadores de la música, quienes laboran en la llamada gig economy. En Chile, esta crisis apareció cinco meses después del inicio de una revuelta social que buscó un cambio estructural del sistema. En este artículo planteamos que esta crisis, conjugada con una politización generalizada en la sociedad chilena, condujo a un resurgimiento del apoyo mutuo entre las y los trabajadores de la música. Ante la sensación de abandono del Estado, la ausencia de políticas culturales adecuadas a las características del trabajo artístico, las y los trabajadores de la música fortalecieron sus organizaciones para ayudarse mutuamente. Desde una perspectiva interdisciplinaria, este artículo discute la revitalización de prácticas y valores de la ayuda mutua entre las y los trabajadores de la música.

Palabras clave: Trabajo artístico, apoyo mutuo, organización social, políticas culturales, precariedad laboral.

\section{Abstract}

The COVID-19 pandemic provoked the cancellation of live music events and other face-to-face musical activities, aggravating the precarious and unstable working conditions of musical laborers, who work within the logics of the gig economy. In Chile, this crisis arrived five months after the beginning of a social movement towards structural changes. This article argues that such a crisis, combined with a political awareness in Chilean society, led to a resurgence of mutual aid between musical workers. Given the state neglect, the absence of cultural policy suitable for artistic work, musical workers strengthened their organizations to help each other. Based on an interdisciplinary approach, we demonstrate the reflourishing practices and mutual aid values between music workers.

Keywords: Artistic work; mutual aid; social organization; cultural policy; precarious working conditions. 


\section{Introducción}

¡Cuántas veces no hemos tenido que poner una firma y unas monedas en las listas de colectas para ayudar a compañeros enfermos, en desgracia o a las familias de fallecidos! Cuántas veces no hemos sentido, también pena y vergüenza al ver que el músico tiene que recurrir poco menos que a la caridad pública! Por suerte, parece, ahora, muy pronto, todo esto va a terminar.

Pablo Garrido (1939, p. 225).

Con la emergencia sanitaria mundial del Covid-19 manifestada en Chile a mediados de marzo de 2020, de la noche a la mañana se suspendieron conciertos y otras actividades musicales presenciales, como talleres y clases, que fueron canceladas o adaptadas al formato en línea. Esto generó un impacto para quienes trabajan en la música en vivo, que, tras la irrupción de internet, ha sido una de las actividades que más ha aumentado sus ingresos (Wikström, 2013). Esto dejó en evidencia la crisis de la institucionalidad cultural estatal chilena, cuyas políticas, planes y programas no se adecuaron a las circunstancias ni a la particularidad del trabajo artístico. Esta crisis, acompañada por una politización generalizada de la ciudadanía a raíz del movimiento social de octubre de 2019 , provocó una sensación de desprotección del Estado frente a la inestabilidad laboral. Ante ello, las y los trabajadores de la música activaron sus propias redes y organizaciones para proveer la ayuda directa que requerían. Así, evidenciamos un resurgimiento del apoyo mutuo entre las y los trabajadores de la música.

Este artículo surge de una investigación sobre el impacto de esta doble crisis en las y los trabajadores de la música en Chile realizada durante el año 2020 en el contexto de la pandemia. Indagamos en las medidas adoptadas por distintos agentes del mundo de la música para enfrentar este escenario, analizando planes y programas implementados por el gobierno chileno en materia de cultura y las acciones desplegadas por organizaciones de trabajadores de la música. El análisis lo realizamos desde la perspectiva del trabajo artístico con un marco teórico y metodológico interdisciplinario y novedoso que conjuga elementos de los estudios de música popular, la historia cultural, la sociología y los estudios del trabajo.

Los resultados presentados buscan contribuir a la emergente literatura académica sobre el trabajo artístico en Chile y, específicamente, sobre el trabajo musical, sumándonos a una discusión más amplia de un marco teórico con ciertos consensos desarrollado por países del norte global. Además, pretendemos visibilizar las condiciones laborales de las y los trabajadores de la música para el diseño de políticas culturales adecuadas a su realidad. El aporte de esta investigación resulta en una de sus limitaciones acerca del carácter emergente de estudios académicos sobre este tema en Chile y América Latina. Por esta razón, esta investigación cualitativa concentra su corpus de análisis en documentos digitales de prensa, sitios web estatales y de gobierno, además de plataformas online y redes sociales oficiales de organizaciones de trabajadores de la música. A su vez, realizamos entrevistas a organizaciones, seleccionadas como estudios de caso por el desarrollo de prácticas de apoyo mutuo.

Nuestro argumento es que, durante la emergencia sanitaria, precedida por una crisis social en el país, las y los trabajadores de la música vivieron un proceso de toma de conciencia sobre sus condiciones laborales y materiales que se expresó en la organización de la ayuda mutua. Este proceso se intensificó frente a la escasa presencia del Estado hacia la protección de sus trabajos y vidas. Comenzaremos analizando las características del trabajo musical, basado en las lógicas de la gig economy, para exponer las condiciones de precariedad a las que quienes trabajan en este campo se enfrentaron a raíz de la crisis social y la emergencia sanitaria. Indagaremos en las políticas culturales dirigidas hacia quienes trabajan en la música a la luz de sus condiciones laborales. Finalmente, delineamos las principales características de la ayuda directa que los mismos trabajadores de la música desarrollaron, para lo cual será fundamental recurrir al concepto de apoyo mutuo, entendido como bidireccional y recíproco. Éste, además, permite la creación de estructuras solidarias para organizar la ayuda mutua. Nuestro estudio evidencia un resurgimiento del apoyo colectivo entre las y los trabajadores de la música junto con una reconocimiento y valoración de su labor como un trabajo. De este modo, 
concluimos que hay una persistencia histórica del apoyo mutuo vinculada a la precariedad y una necesidad de pensar críticamente las políticas, planes y programas culturales en relación a ella.

\section{El trabajo musical}

El trabajo musical se caracteriza por su lógica de gig economy, que traducimos como la economía de la tocata. En este estudio concebimos como trabajadores de la música a músicos y a las y los trabajadores de la cadena productiva del mundo de la música. Con músicos nos referimos a todas aquellas personas trabajando o "buscando empleo remunerado en el campo musical" (Williamson \& Cloonan, 2016, p. 8)'. Por razones metodológicas, no consideramos a aquellas que hacen música por placer y sin pretensiones de generar ingresos. Estos trabajadores desarrollan su labor de manera independiente, sin contrato o, como mucho, con contratos de palabra o estacionales. Por esta razón, no tienen un ingreso estable, sino que éste depende del número y la recurrencia de actividades que realicen, gozando muy pocos de ellos seguridad social.

Esta característica del trabajo musical no es exclusiva al contexto chileno ni al tiempo contemporáneo. Por el contrario, como se ha planteado ampliamente, el trabajo musical es de naturaleza independiente e informal. De hecho, se acuñó el término the gig economy para referirse a trabajos independientes o de corto plazo, donde los trabajadores se sustentan con diversos trabajos de tiempo parcial que no proveen beneficios propios de empleos tradicionales, como seguro de salud o previsión social (Hook, 2015). Un ejemplo son los empleos que ofrecen empresas como Uber, subcontratando a trabajadores por períodos cortos, en vez de puestos a tiempo completo (Cloonan \& Williamson, 2017, p. 493). Lo interesante aquí, como plantean Cloonan y Williamson, es que este concepto comenzó a ser usado para describir una situación laboral considerada precaria y de explotación que no es exclusiva del ámbito musical, pero cuyo nombre proviene de él. Esto nos lleva a reflexionar acerca de las condiciones laborales de los músicos.

Aunque el uso de este concepto en el mundo de la economía sea relativamente nuevo, las nociones de precariedad y de trabajo inmaterial en labores artísticas son de larga data, pues los músicos han trabajado en la gig economy lantes de que se denominara así) "durante la mayor parte del siglo [veinte] y la naturaleza de su trabajo no ha sido tomada en consideración" (Cloonan \& Williamson, 2017, p. 494). De manera similar, sobre la base de su estudio del trabajo musical en Grecia, Tsioulakis argumenta que éste "siempre ha sido precario", y, por lo mismo, mucho antes del período de austeridad, los músicos "ya tenían herramientas para enfrentar la crisis" (2020a, p. 164). Siguiendo a Tsioulakis, sostenemos que la experiencia de las y los trabajadores de la música en este período de crisis, nos dará luces sobre cómo proteger las industrias culturales, especialmente a sus trabajadores (2020b).

En Chile, las y los trabajadores de la cultura (creadores, intérpretes, gestores y técnicos) laboran en un contexto de precariedad de base: un $88,3 \%$ trabaja sin contrato y un $59 \%$ no está afiliado a ningún sistema de pensiones ni de seguridad social (Brodsky, Negrón \& Possel, 2014, p. 24, 35). Para los trabajadores de las artes performativas, como la música, esta situación es más problemática, porque su trabajo rara vez se traduce en un producto material fácilmente medible. Esto implica una dificultad para generar ingresos asociados al resultado del trabajo y complejiza la valoración económica de la labor artística. Esto último está ligado al antiguo problema del arte como trabajo (Becker, 1982; Goehr, 1992) y, en particular, a la dificultad de concebir a los músicos como trabajadores (Kraft, 1995; Stahl, 2013). Además, la idea de que la música es resultado del talento, el goce y la genialidad individual más que del esfuerzo (Miller, 2008), genera autoexplotación laboral (Gross \& Musgrave, 2020), trabajo no remunerado (Brook, O'Brien \& Taylor, 2020) y otras inequidades.

\section{De una crisis social a una crisis sanitaria: "llegó la hora de reinventarse"}

Las manifestaciones sociales iniciadas el 18 de octubre de 2019 evidenciaron las tareas pendientes del proceso de democratización de los últimos treinta años. Uno de los principales malestares expresados en las calles fue la profundización del 
programa neoliberal instalado durante la dictadura cívico-militar de Pinochet (Riethof, 2019). La magnitud de la protesta impactó también la vida cotidiana y el desarrollo de las actividades urbanas, como la música en vivo.

A nivel global, el punto de quiebre que generó la descarga masiva de archivos musicales y audiovisuales en la década de los 2000 llevó a las grandes corporaciones discográficas a repensar el lugar de la inversión en la cadena de producción musical. En Chile, la realización de eventos en vivo se ha constituido como un sector rentable de inversión y ha pasado a ser una importante fuente laboral (ODMCh, 2020, 12-13). En el marco post-revuelta social del 18 de octubre de 2019 se cancelaron eventos masivos, como festivales, conciertos, fiestas de año nuevo y cerraron temporalmente bares, restaurantes, centros culturales y educativos que forman parte del circuito laboral de la música en vivo, reduciendo las opciones laborales. Incluso, bandas musicales ya legendarias como los integrantes de la Sonora de Tommy Rey - que han dedicado toda su vida adulta a la música en vivo-, a solo un mes del estallido social plantearon que había llegado "la hora de reinventarse" a causa de la cancelación de sus conciertos (CNN, 2020). Este panorama se agudizó con la llegada de la crisis sanitaria.

La venta de boletos de espectáculos de música en vivo entre 2018 y 2019, como efecto de la crisis social, bajó en un $22,9 \%$ (ODMCh, 2021). La pandemia tuvo un impacto aún mayor, cayendo drásticamente la venta de entradas en un $76,5 \%$ durante 2020 . La combinación de ambas crisis condujo a un desplome del $86,7 \%$ en la venta de entradas (ODMCh, 2021). La imposibilidad de realizar conciertos con público en vivo y/o presencial, impactó profundamente en los ingresos de músicos y técnicos, así como en sus condiciones materiales (ODMCh, 2020, pp. 20-30). Después de una primavera interrumpida por las movilizaciones sociales de 2019, el estado de excepción y el arribo de la pandemia el último mes del verano de 2020, la precariedad ya característica del sector se agudizó exponencialmente (ODMCh, 2020). Y, sobre todo, si consideramos que la informalidad en las y los trabajadores de la música es de un $89 \%$ (ODMch, 2020) y en un país donde los trabajadores informales históricamente han sido outsiders de la distribución de bienes y servicios de las políticas sociales (Farías, 2019).

\section{Las políticas culturales hacia los trabajadores de la música}

La conceptualización teórica de política cultural busca destacar su pluralidad, pues no pueden ser reducidas sólo a la acción gubernamental. Hay múltiples actores involucrados en su formulación, mediación y recepción (García Canclini, 1987), los roles que asumen los agentes directos de los circuitos culturales (mercado, administración pública o comunidad) también son plurales (Brunner, 1987) o bien, pueden ser un puente desde el soporte de las instituciones entre los registros estético y antropológico (Miller \& Yúdice, 2004). De hecho, como define la etnomusicóloga Ana María Ochoa, la política cultural es en efecto "la movilización de la cultura llevada a cabo por diferentes tipos de agentes - el Estado, los movimientos sociales, las industrias culturales, instituciones tales como museos u organizaciones turísticas, asociaciones de artistas y otros- con fines de transformación estética, organizacional, política, económica y/o social" (2003, p. 20).

En esta sección, exploramos las acciones tomadas por el Estado de Chile en las últimas décadas, desde la política cultural, hacia los trabajadores de la música. Analizaremos el diálogo que la sociedad civil ha realizado con la institucionalidad cultural, especialmente en el contexto de la doble crisis. La política cultural del Estado chileno, a pesar de querer desligarse de su pasado dictatorial de censura, promoviendo espacios de reparación simbólica tras la violación sistemática a los derechos humanos, se caracteriza por su perfil subsidiario y de concursabilidad (Peters, 2020). Así, el modelo de Estado del proyecto neoliberal que se caracteriza por el repliegue de su accionar y el traspaso de gran parte de sus responsabilidades a entes privados, mediante la tercerización de servicios, también impacta en el mundo de la cultura. Esto apunta al florecimiento de una industria cultural próspera y competitiva, en la cual el principal apoyo a las y los trabajadores de la cultura se hace de manera indirecta mediante concursos de fondos públicos, lo que afecta la continuidad de proyectos y horada cualquier opción de estabilidad laboral y de trabajo digno (OPC, 2014, p. 219).

En este sentido, con la doble crisis, social y sanitaria, las acciones estatales no dieron abasto con un Plan Económico de Emergencia basado en bo- 
nos que no cubrían las necesidades básicas de los grupos familiares más vulnerables ${ }^{2}$. La escasa presencia del Estado frente a la crisis que experimentaron las y los trabajadores de la cultura, en general, y de la música, en particular, activó campañas y petitorios que distintas organizaciones del sector presentaron durante la emergencia sanitaria, las cuales analizaremos en la siguiente sección ${ }^{3}$.

Entre las iniciativas internacionales desarrolladas por los Estados para ayudar a los trabajadores de la cultura a enfrentar la crisis, han existido ejemplos de ayudas materiales directas (Platea Magazine, 2020; BAE Negocios, 2020). En el caso chileno, sólo luego de que los gremios de trabajadores de la cultura elevaran su voz denunciando la urgencia del problema (Becerra, 2020) y exigiendo al gobierno disponer de fondos (Solo Artistas Chilenos, 2020), el Ministerio de la Cultura, las Artes y el Patrimonio (MINCAP) abrió un nuevo concurso de fondos de emergencia para la generación de contenidos virtuales. Si bien éstos se traducirían en una ayuda concreta para algunos, respondieron a la misma lógica subsidiaria, de concursabilidad y tercerización de servicios de las instituciones culturales del Estado (Olmedo, 2020).

El carácter de estos fondos de emergencia, en vez que fueran de acceso directo a los que cualquier trabajadora o trabajador de la cultura postulase por perfil socioeconómico o cesantía -como solicitaban las organizaciones de músicos ISINAMUARCHI, 2020)-, fueron entregados por calidad y pertinencia de los proyectos postulantes. Otro problema que observamos fue el escaso monto que podían destinar los proyectos por concepto de honorarios por persona (un máximo de $\$ 300.000$ ). En la convocatoria del Consejo de Fomento a la Música Nacional también consideramos problemática la separación de los postulantes por género musical, ofreciéndole un monto máximo de $\$ 448.180$ a quienes compusieran obras clásicas o doctas, mientras que por una obra de carácter popular o raíz folclórica, un máximo de $\$ 336.134$. En los concursos anteriores del MINCAP, estos dos últimos géneros figuraban separados y ahora competían por un mismo fondo, gozando los doctos del privilegio de competir entre ellos (MINCAP, 2020).

Estos fondos de emergencia no subsanaron el problema de necesidad de apoyo hacia las y los trabajadores de la cultura. Por una parte, existió una muy baja tasa de postulación ${ }^{4}$ y una evaluación insuficiente - un 2,4 en una escala de 1 a $7-$ con la que las y los trabajadores de la cultura calificaron las ayudas del MINCAP. En este sentido, constatamos que no solo fue evidente la insuficiencia del plan de apoyo hacia las necesidades del sector, sino también su desconexión con la realidad material y laboral de las y los trabajadores culturales (OPC, 2021).

La particularidad de las condiciones laborales de las y los trabajadores de la cultura, al responder a las lógicas de la gig economy, significó que el $54 \%$ no recibiera ninguna de las medidas de apoyo que destinó el gobierno a los trabajadores y las familias en general (OPC, 2020), porque no cumplían los requisitos para solicitarlas ${ }^{5}$. Por el contrario, la mayoría (un 59,3\%) recurrió a sus propios ahorros o recibió ayudas de familiares, amigos o vecinos (OPC, 2021).

Consideramos que la pandemia, al llegar a Chile a meses del estallido social, se encontró con una ciudadanía empoderada y organizada, y, al mismo tiempo, con trabajadores de la música que se desempeñan de manera independiente, a honorarios, por lo que son muy sensibles a momentos de crisis. En este contexto, surgieron y se consolidaron redes de apoyo desde la sociedad civil frente a la desprotección estatal. Al no encontrar apoyo en las instituciones estatales, las y los trabajadores de la música develaron con su activismo una vez más “la precaria situación histórica de la industria musical chilena" (IMUVA, 2020b).

\section{Ayuda mutua como respuesta a la crisis: antecedentes y resurgimiento}

En esta sección afirmamos que, al encontrar insuficientes las medidas implementadas por el Estado en un contexto de catástrofe mundial y crisis económica, las y los trabajadores de la música recurrieron a sus propios pares, articulando redes de ayuda mutua, donde primó el tejido y el afianzamiento de lazos comunitarios desde la virtualidad y la acción concreta de ayuda material. En su estudio de las catástrofes de los siglos XX y XXI, como la pandemia del Covid-19, Rebecca Solnit (2020) muestra cómo de éstas emergen comu- 
nidades fortalecidas. La sociedad civil prioriza la organización social y el bien común que, a través de la creatividad y el acopio de recursos, se materializan en acciones generalizadas de altruismo y ayuda mutua para enfrentar los desafíos. Este tipo de acciones se pueden rastrear desde la publicación de Peter Kropotkin (1902) en que conceptualiza la noción de apoyo mutuo como una alternativa al darwinismo social de la segunda mitad del siglo XIX, proponiendo que la solidaridad y la cooperación son las mejores armas para la sobrevivencia. Con esto demostraba que la garantía para la evolución de la humanidad es la práctica de la ayuda mutua y no la lucha mutua ni la competencia (Kropotkin, 1902).

La ayuda mutua tuvo expresión desde mediados del siglo XIX con la expansión del anarquismo y el movimiento mutualista. En Chile las y los trabajadores comienzan a formar sociedades de resistencia y de socorro mutuo en el marco de la llamada "cuestión social", cuando aún no existían leyes de seguridad social, ni ningún tipo de protección legal para los trabajadores. Si bien había importantes diferencias ideológicas entre estas organizaciones, eran de base comunitaria y solidaria. Primero, organizaron la ayuda mutua buscando el bienestar físico de sus miembros, para luego extender su alcance a intereses colectivos, como el desarrollo intelectual y el cambio social ${ }^{6}$.

A lo largo del siglo XX, los valores, ideologías y formas de las organizaciones de trabajadores mutaron según las circunstancias sociales y económicas. También han cambiado las nociones sobre el trabajo en cultura, alcanzando en el último tiempo una mayor aceptación social como una labor que debe ser remunerada y a sus trabajadores, concebidos como sujetos de derecho (Pinochet, 2020). Sin embargo, la persistencia histórica de la precarización del cuidado del cuerpo de las y los trabajadores, por ser considerados sólo una fuerza de trabajo ha sido una constante (Illanes, 2020). En Chile, en los últimos treinta años, a pesar de la implementación del modelo neoliberal, el quiebre del tejido social heredado de la dictadura y los cambios en la industria musical, existen experiencias formales de asociatividad entre las y los trabajadores de la música, pero sin la "capacidad de aglutinar a todos los músicos y menos de hacer valer sus derechos frente a los sectores público y privado" (Karmy, Brodsky, Facuse \& Urrutia, 2015, pp.19-20). Entre ellas encontramos sindicatos, como el SINAMUAR$\mathrm{CHI}$ (Sindicato de Músicos y Artistas de Chile); gremios como la Unión Nacional de Artistas (UNA) en la que participan músicos; la Sociedad Chilena del Derecho de Autor (SCD) 2015 que, además de gestionar derechos, ha asumido un rol gremial frente a instancias de negociación política. También existen organizaciones informales para establecer redes de colaboración local, nacional o producir festivales y conciertos ${ }^{7}$. Observamos una alta capacidad organizativa con fines creativos y la gestión de derechos de autor, mas una baja asociatividad con fines gremiales y laborales lo que dificulta una mejora en las condiciones laborales de las y los trabajadores de la música (OPC \& IMICHILE, 2016).

Con la doble crisis, surgieron nuevas iniciativas que apuntaron a visibilizar y mejorar las condiciones económicas de las y los trabajadores de la música. Entre ellas, Managers Asociados de Músicos en Chile (MAMCHI) inició la campaña \#MásMúsicaChilena en abril de 2020, para difundir producciones locales. De manera similar, el Gremio de la Industria Musical de Valparaíso (IMUVA) creó playlists de música chilena en plataformas streaming para difundir a artistas nacionales y preparó una guía sanitaria para realizar conciertos online de manera segura (IMUVA 2020a). En mayo de 2020, emerge la Asociación Gremial de Trabajadores de las Artes y Espectáculos (AGTAE) para apuntar directamente a la crisis, organizando talleres en línea e informando sobre las ayudas estatales. AGTAE se sumó también a la campaña internacional \#AlertaRoja con una intervención pública en Santiago en septiembre de 2020.

Otras acciones buscaron generar ingresos directos para las y los trabajadores del sector. Entre ellas, algunas instituciones culturales tanto públicas, privadas y de gestión privada con financiamiento público, decidieron mantener su programación musical anual, pero desde plataformas virtuales o, bien, ofreciendo sus plataformas para que los mismos músicos realizaran talleres y conciertos en línea. A su vez, la SCD ofreció repartir mil millones de pesos entre sus asociados, dependiendo de los derechos generados por cada socio (El Mostrador, 2020).

Estas iniciativas ayudaron a sortear la agudización de la precarización laboral de las y los trabajadores de la música, sensibilizando al público de la situa- 
ción y generando ayudas materiales. Sostenemos que las y los trabajadores de la música afectados por la doble crisis, al observar un apoyo escaso por parte de la institucionalidad cultural del Estado, gestionaron iniciativas de apoyo mutuo, dando pie a una revitalización de estas prácticas solidarias, horizontales y recíprocas de larga data. La autogestión adquirió, entonces, un nuevo protagonismo para cubrir necesidades de subsistencia como alimentos y mercadería, y organizada bajo las lógicas del apoyo mutuo.

\subsection{Estudios de caso de apoyo mutuo para ayudas directas: CONATEMUCH y TRAMUS}

En esta sección analizamos dos organizaciones que gestionaron acciones para proveer ayuda directa y material a las y los trabajadores de la música en el contexto de la doble crisis. Las acciones fueron gestionadas desde la solidaridad y la organización de base voluntaria y demuestran un fortalecimiento del tejido social y una revitalización del apoyo mutuo frente a la escasa protección estatal hacia el mundo de la cultura.

La Corporación Nacional del Teatro Musical Chileno (CONATEMUCH) es una organización no gubernamental formada el año 2009 que busca promover el "desarrollo, especialmente de personas, familias, grupos y comunidades que viven en condiciones de pobreza y/o marginalidad" (CONATEMUCH, 2020b). Sus actividades abarcan ámbitos de educación, cultura, trabajo, entre otras, y consideran el teatro musical como un medio de transformación social (2020b). Sus miembros realizan labores relacionadas con el teatro musical, en tanto intérpretes, compositores, técnicos, bailarines, productores y directores, entre otros. En 2020, contaba con 100 afiliados y/o asociaciones afiliadas con mil artistas en todo Chile. Sus referentes son el Sindicato de Actores y Actrices de Chile (SIDARTE) y la SCD, y relevan la coordinación con organizaciones como la SCD, la UNA y la Sociedad de Directores Audiovisuales, Guionistas y Dramaturgos, porque desde "ahí se generan las políticas públicas" (Soto, 2020). Con éstas consolidaron sus redes de trabajo frente a la doble crisis. CONATEMUCH lleva un tiempo cabildeando para ser reconocida en la Ley de Artes Escénicas (Ley 21.175) y, también, por la contingencia, participan en la CICE dialogando con el gobierno para el "bienestar de los artistas en general" (Soto, 2020).

En esta situación, miembros de CONATEMUCH consideraron necesario realizar acciones de ayuda mutua por el grave impacto laboral de la pandemia. En asamblea, fue acogida la moción para iniciar la campaña "Es hoy por ti, mañana por mí" (Figura 1) que reunió alimentos no perecibles y/o dinero para ayudar directamente a los trabajadores del teatro musical que "han perdido trabajos y [...] en consecuencia no tienen dinero para alimentarse" (CONATEMUCH, 2020a). En otras palabras, para otorgar un apoyo a la subsistencia biológicacorporal de sus integrantes y, al mismo tiempo, apelando al valor de la reciprocidad y la solidaridad de clase, visible en el lema de la campaña: "Solo el pueblo ayuda al pueblo" (Figura 1). Además, frente a esta situación, existió un mayor interés de las y los integrantes por participar en instancias de discusión de la labor gremial, facilitado por la mayor periodicidad de las asambleas, ahora en formato virtual, con lo que articularon nuevas comisiones de trabajo leducación, comunicaciones y ley de artes escénicas). Estas transformaciones han descentralizado el trabajo de representación que antes recaía en el directorio.

Figura 1. Afiche de la campaña "Es hoy por ti mañana por mí" de CONATEMUCH.

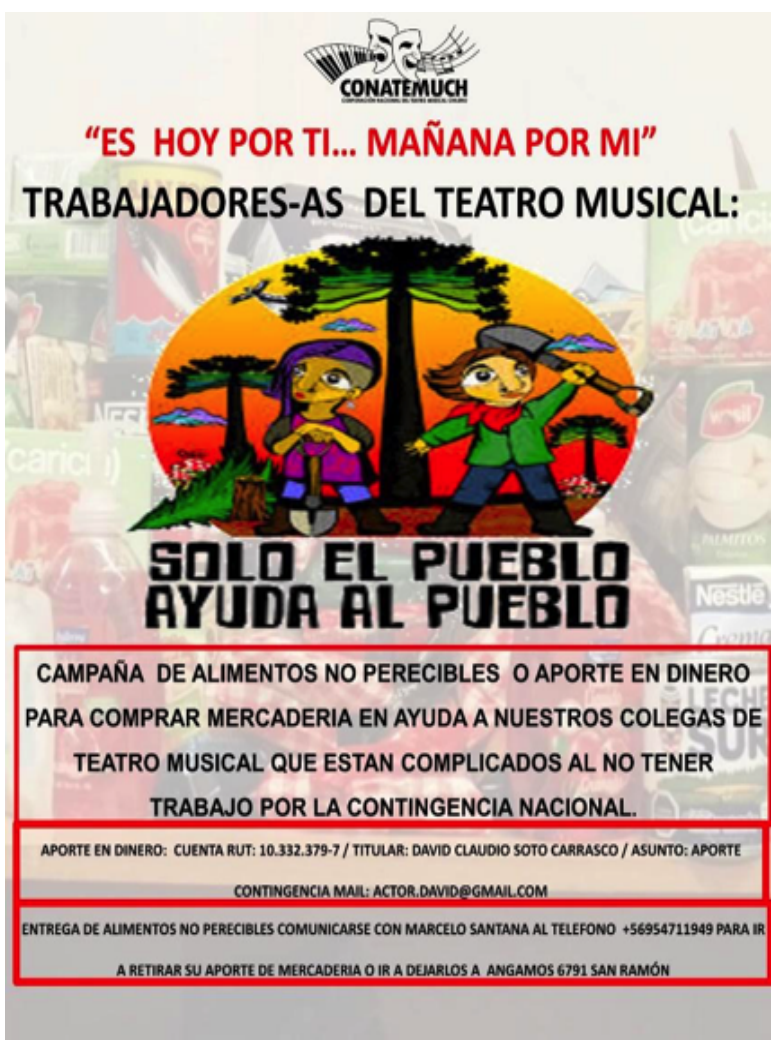

Fuente: Facebook CONATEMUCH. 
La segunda organización es Trabajadoras de la Música, Mujeres y Disidencias (TRAMUS), red transfeminista autoconvocada que se organizó para la marcha del 8 de marzo de 2019 para proveer apoyo, contención y organizar activismo político. Desde mayo de 2020 ha sido una de las interlocutoras con el Estado en el Consejo de la Música, presentando su agenda de género para el sector musical (El Desconcierto, 2020). A diferencia de CONATEMUCH, TRAMUS es una red informal, sin personalidad jurídica, a la que puede unirse cualquier mujer o disidencia que trabaje en el sector musical y adscriba al manifiesto publicado en su sitio web (Videla, 2020). Su objetivo es promover la organización colectiva de mujeres y disidencias en la música, con un enfoque feminista, enfatizando en la sororidad y está compuesta por "todxs quienes se desempeñan en el ámbito de la música" (TRAMUS, 2019). TRAMUS ganó visibilidad con las movilizaciones de octubre de 2019, participando en manifestaciones y adscribiendo a las demandas de la ciudadanía.

Con la emergencia sanitaria, publicaron comunicados haciendo un llamado a la solidaridad y a “actuar en conjunto", ya que, como se demostró el 18 de octubre, es lo que "nos hace más poderoses en estas situaciones" (TRAMUS, 2020a). Interpelaron al MINCAP para escuchar a los sectores que representan la cultura y a tomar medidas “suficientes para subsanar y proteger a trabajadores/ trabajadoras" (TRAMUS, 2020a). Hicieron circular un petitorio con medidas en relación a la contingencia sanitaria y la necesidad de "una mesa de diálogo para abordar, desde el enfoque de género, un plan de medidas de emergencia para el sector musical" (TRAMUS, 2020b). Tras la realización de una encuesta para conocer el impacto de la pandemia en la red, pusieron en marcha un plan de ayuda directa al que llamaron Plan SOS. Este plan consistió en repartir canastas de mercadería entre sus integrantes. Éstas fueron financiadas desde la autogestión y ensambladas a partir del trabajo voluntario con campañas como “lucatón para las canastas" y "aprende junto a TRAMUS", con talleres y cursos en línea, impartidos por las mismas personas asociadas a la red, reuniendo un fondo colectivo, bajo el lema "entre todes nos cuidamos" (TRAMUS, 2020c; Figura 2). La reciprocidad a la que apela este lema y la lógica del fondo colectivo, hacen eco de las antiguas sociedades de resistencia y las mutualistas, organizaciones que, al administrar un fondo común y autónomo, se constituían como sujetos autónomos y soberanos (Salazar, 2012).

Figura 1. Afiche campaña “Aprende junto a TRAMUS".

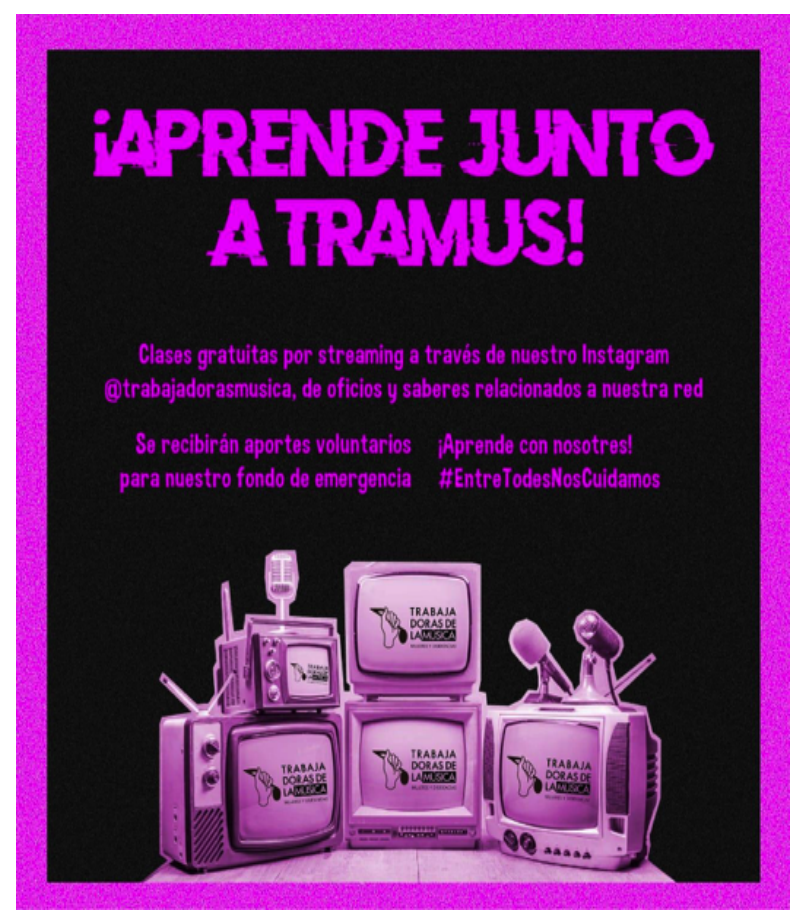

Fuente: Facebook TRAMUS

Si bien ninguna de estas organizaciones explicitó un vínculo de sus prácticas de colaboración con las organizaciones de trabajadores y músicos del pasado, es interesante observar cómo, a pesar de esa aparente desconexión, existen continuidades en las prácticas de ayuda ancladas en la memoria social que resurgen en tiempos de crisis llllanes, 2020). Lo novedoso de estas dos campañas es, sin embargo, la noción de "trabajadores" al centro de sus demandas, ampliando la conceptualización de lo que significa trabajar en música, incluyendo a todos quienes trabajen en oficios y profesiones involucrados en su cadena productiva. Otra novedad de estas campañas la constituye su agenda política para incidir en la política cultural, como la inclusión en la Ley de Artistas en el caso de CONATEMUCH, y la agenda feminista, en el caso de TRAMUS. Y, por último, además de una noción de solidaridad gremial, específicamente en el caso de TRAMUS, hay también una solidaridad entre mujeres y disidencias sexuales, poniendo la sororidad al centro como un valor aglutinador y de motor de cambio. 
La revitalización del apoyo mutuo viene con nuevas nociones sobre el trabajo musical y un enfoque de género que proyecta una transversalidad que se condice con un interés político que va más allá del netamente gremial.

\section{Conclusión}

En este artículo planteamos que la pandemia develó y sigue profundizando tanto la precariedad de las y los trabajadores de la música en Chile, como el abandono del Estado, asuntos que ya venían discutiéndose durante el proceso de concientización anti-neoliberal del movimiento de octubre. Analizamos las condiciones laborales de las y los trabajadores de la música desde una perspectiva interdisciplinaria, destacando la informalidad y la independencia como los principales elementos que le dan forma a la llamada gig economy. Planteamos que, con la irrupción de la crisis social de octubre 2019, el volumen de trabajo en la música disminuyó, lo que se agudizó con la cancelación de espectáculos en vivo debido a la pandemia a partir de marzo de 2020. Al mismo tiempo, analizamos las políticas culturales que implementó el Estado en el contexto de la doble crisis, develando cómo éstas no se ajustaron a la realidad material de las y los trabajadores de la música ni a la naturaleza de su trabajo.

Demostramos cómo la combinación de la doble crisis mostró una reactivación de la organización entre trabajadores de la música que desarrollaron campañas como la promoción de la escucha de música chilena, colectas de dinero y entrega de alimentos. Estas campañas fueron lideradas por organizaciones autogestionadas y autofinanciadas, que surgieron o tomaron fuerza con la revuelta de octubre. Los estudios de caso evidenciaron la revitalización de la ayuda mutua entre las y los trabajadores de la música, con la gestión de ayudas directas de base horizontal fundamentadas en los valores de solidaridad gremial y de género, apostando al cambio social y la incidencia en la política cultural ¿Podrán constituirse estas redes de solidaridad en un nuevo actor social frente a la institucionalidad cultural chilena, para promover la valorización social hacia la labor de los trabajadores de la música?
El trabajo musical se ha caracterizado por su precariedad desde mucho antes que se conjugara esta doble crisis. Por ello, sostenemos la importancia de no retomar una normalidad que ha sido largamente desigual e injusta para las y los trabajadores de la música. Por el contrario, es necesario priorizar cómo diseñar una política pública que asegure condiciones laborales dignas, con un pago equitativo y el resguardo de los derechos sociales para las y los trabajadores de la música en el país.

\section{Notas}

1. Esta y todas las traducciones al castellano de citas publicadas originalmente en otros idiomas son de las autoras.

2. Una iniciativa fue el Bono COVID, de $\$ 50.000$ por cada beneficiario (alrededor de USD \$70) o el Ingreso Familiar de Emergencia, que consistió en una entrega de dinero durante tres meses (Ministerio Secretaría General de Gobierno, 2020)

3. La cesantía en las actividades artísticas, de entretenimiento y recreativas ocupó el segundo lugar del sector más golpeado por el desempleo (Molina 2020).

4. El Monitoreo Nacional de Trabajadores de la Cultura, quienes en su mayoría trabajaban en música arrojó en junio que el $76 \%$ de los encuestados no postuló a los fondos de emergencia del MINCAP (OPC, 2020, p. 22). En octubre de 2020, la cifra cambió a un $61 \%$ que no postuló (OPC, 2021). Llama la atención el bajo porcentaje de postulación, considerando que un $81 \%$ vio disminuida su principal actividad laboral cultural o dejó de trabajar en ella y un $69 \%$ tuvo una baja en sus ingresos (OPC, 2020).

5. Aquellas medidas sociales a las que accedieron los trabajadores de la cultura, en un primer momento, fue la devolución anticipada de impuestos y la caja de alimentos (22\% y $17 \%$ respectivamente) (OPC 2020). En un segundo momento, un $22,7 \%$ accedió al Ingreso Familiar de Emergencia, un 20,5\% al aporte fiscal para la clase media y un $18,9 \%$ a préstamos de la ley de protección de los trabajadores independientes (OPC, 2021, 3). Estas ayudas estaban destinadas a las y los trabajadores independientes en general.

6. La fundación de la Sociedad de la Igualdad (1850) fue relevante en la materialización de estos valores. En el caso de los músicos, en 1889 se crea la Sociedad Musical de Socorros Mutuos de Santiago (Karmy, 2019).

7. Por ejemplo, las organizaciones de trabajadores constituidas tras los procesos de formación del programa estatal Escuelas de Rock (Urqueta, 2019) o los festivales La Matria Fest y Ruidosa Fest. 


\section{References}

Becker, H. S. (1982). Art worlds. Los Angeles: University of California Press. London.

Becerra, A. (19 marzo, 2020). Mario Rojas: “Existe un clima de desamparo con los artistas”. Diario Uchile. Recuperado de https://radio.uchile.cl/2020/03/19/mario-rojas-existeun-clima-de-desamparo-total-con-los-artistas/.

Brodsky, J., Negrón, B., \& Pössel, A. (2014). El escenario del trabajador cultural en Chile. Artes visuales, artes escénicas, literatura, música, audiovisual. Proyecto Trama/ Observatorio de Políticas Culturales. Recuperado de https://ec.cultura.gob.cl/wp-content/ uploads/2020/10/0_50_El-Escenario-del-Trabajador-Cultural-en-Chile.pdf

Brook, O., O'Brien D. \& Taylor, M. (2020). Culture is Bad For you. Inequality in the cultural and creative industries. Machester University Press.

Brunner, J. (1987). Políticas culturales y democracia: hacia una teoría de las oportunidades. En N. García Canclini (Ed.), Políticas Culturales en América Latina (pp. 1750203). Cultura y Sociedad.

Cloonan, M. \& Williamson, J. (2017). Introduction. Popular Music and Society, 40(5), 493-498. https://doi.org/10.1080/03007766.2017.1351117

CNN. (27 noviembre, 2020). “Tommy Rey por cancelación de eventos: 'Llegó la hora de reinventarse porque la cosa no está fácil'”. Recuperado de https://www.cnnchile.com/ cultura/tommy-rey-por-cancelacion-de-eventos_20191127/

CONATEMUCH. (18 marzo, 2020a). Es hoy por ti mañana por mí. [Imagen adjunta]. [Actualización de estado]. Facebook. https://www.facebook.com/1278252442272993/photos/ a.1278262918938612/2727247637373459/?type=3\&theater

CONATEMUCH. (3 agosto, 2020b). CONATEMUCH. [Imagen adjunta]. [Actualización de estado]. Facebook. https://web.facebook.com/TeatroMusicalChileno/photos/pcb.306215999 $7215553 / 3062159873882232$

El Desconcierto. (22 mayo, 2020). “Red de Trabajadoras de la Música participa por primera vez en el Consejo de la Música". Recuperado de https://www.eldesconcierto.cl/tiposmoviles/2020/05/22/red-de-trabajadoras-de-la-musica-participa-por-primeravez-en-el-consejo-de-la-musica.html

El Mostrador. (14 abril, 2020). “SCD repartirá $\$ 1.000$ millones entre sus socios y afiliados por la crisis del coronavirus". Recuperado de https://www.elmostrador.cl/cultura/2020/04/14/scd-repartira-1-000-millones-entre-sus-socios-y-afiliados-por-lacrisis-del-coronavirus/?fbclid=IwAR1elOayrJZTJFHBxr7_zYhZuCM11BksTEVPjAQNZozRrWng6hDNTsnVMvU.

Farías, A. (2019). Políticas sociales en Chile: Trayectoria de inequidades y desigualdades en distribución de bienes y servicios. Ediciones Universidad Alberto Hurtado.

García Canclini, N. (1987). Introducción. Políticas culturales y crisis de desarrollo: un balance latinoamericano. En N. García (Ed.), Políticas culturales en América Latina (pp. 1361). Cultura y Sociedad.

Garrido, P. (10 noviembre, 1939). Descanso para el músico. Lo que debe saber el público. Una justa petición de los músicos. Las Últimas Noticias, p. 225.

Goehr, L. (1992). The imaginary museum of musical works: An essay in the philosophy of music. Clarendon Press.

Gross, S.A \& G. Musgrave. (2020). Can Music MakeYou Sick? Measuring the Price of Musical Ambition. University of Westminster Press. 
BAE Negocios. (20 marzo, 2020). “Habrá fondos para que la cultura pueda sobrevivir al aislamiento". Recuperado de https://www.baenegocios.com/cultura/Habra-fondospara-que-la-cultura-pueda-sobrevivir-al-aislamiento-20200320-0037.html.

Hook, L. (29 diciembre, 2015). Year in a Word: Gig Economy. Financial Times online. Recuperado de https://www.ft.com/content/b5a2b122-a41b-11e5-8218-6b8ff73aae15/

Illanes, M. (9 julio, 2020). Núcleo Historia Social Popular y Autoeducación Popular de la Universidad de Chile. Primera conversación pandémica con María Angélica Illanes. [video adjunto]. [Actualización de estado]. Facebook. https://www.facebook.com/ watch/live/?v=273039510574458\&ref=watch_permalink.

IMUVA. (25 abril, 2020a). Guía sanitaria enseña a realizar transmisiones musicales vía streaming de forma segura. Recuperado de http://imuva.cl/guia-sanitaria/

IMUVA. (20 agosto, 2020b). Declaración de la Red de Asociaciones de Música Chilena ante la crisis sanitaria y económica que afecta al sector. Recuperado de http://imuva.cl/ declaracion-de-red-de-asociaciones-de-musica-chilena-ante-la-crisis-sanitariay-economica-que-afecta-al-sector-2/.

Karmy, E., Brodsky, J., Facuse, M. \& Urrutia, M. (2015). El papel de las políticas públicas en las condiciones laborales de los músicos en Chile. CLACSO. Recuperado de http://biblioteca.clacso.edu.ar/clacso/becas/20150520052544/ElPapelDeLasPolitciasPublicas. pdf.

Karmy, Eileen. 2019. “The Path to Trade Unionism: Musical Work in Chile (1893-1940)” Tesis de doctorado. Universidad de Glasgow. Recuperada de http://theses.gla.ac.uk/75178/

Kraft, J. P. (1995). Artists as Workers: Musicians and Trade Unionism in America, 1880-1917. The Musical Quarterly, 79(3), 512-543. http://www.jstor.org/stable/742442

Kropotkin, P. (1902). Mutual aid: a factor of evolution. McClure Phillips \& Co.

Miller, K. H. (2008). Working musicians: Exploring the rhetorical ties between musical labour and leisure. Leisure Studies, 27(4), 427-441. https://doi.org/10.1080/02614360802334963

Miller, T., \& Yúdice, G. (2004). Política Cultural. Gedisa.

MINCAP. (2020). Línea de circulación y creación artística. Fondo de la Música. Ministerio de las Culturas, las Artes y el Patrimonio. Recuperado de . https://www.fondosdecultura. cl/linea-de-circulacion-y-creacion-artistica-fondo-de-la-musica/.

Ministerio Secretaría General de Gobierno. (2020). Apoyo a los ingresos de las familias. Recuperado de https://www.gob.cl/planeconomicoemergencia/apoyo/.

Molina, T. (10 junio, 2020). Encuesta UC: Tasa de desempleo en mayo sube hasta un 11,2\% a nivel nacional por crisis sanitaria. Emol. Recuperado de https://www.emol.com/ noticias/Economia/2020/06/10/988696/Tasa-desempleo-UC-112.html

Ochoa, A. (2003). Arenas movedizas: arte, cultura, política. ICANH.

ODMCh. (2020). Diagnóstico de la Industria Musical Chilena. Estallido Social y Covid-19. Observatorio Digital de la Música Chilena. Recuperado de https://www.api.odmc.cl/DiagnosticodelalndustriaMusicalChilena.EstallidoSocialyCOVID-19(7).pdf

ODMCh. (2021). Informe de caracterización de la industria musical chilena. Hacia un ecosistema de la música. Capítulo 2: Música en vivo. Observatorio Digital de la Música Chilena. Recuperado de https://www.api.odmc.cl/Maestra_ODMC_CAP2_VF.pdf

Olmedo, C. (8 mayo, 2020). El acelerador neoliberal en las políticas culturales "anticrisis". El Mostrador. Recuperado de https://www.elmostrador.cl/cultura/2020/05/08/elacelerador-neoliberal-en-las-politicas-culturales-anticrisis/

OPC. (2014). El escenario del trabajador cultural en Chile. Santiago de Chile: Proyecto Trama, Observatorio de Políticas Culturales. Recuperado de http://www.observatoriopoliticasculturales.cl/publicaciones/el-escenario-del-trabajador-cultural-en-chile/ 
OPC. (2020). Monitoreo nacional trabajadores de la cultura. Primeros resultados, septiembre 2020. Observatorio de Políticas Culturales. Recuperado de http://www.observatoriopoliticasculturales.cl/wp-content/uploads/2020/09/primerosresultadosmonitoreo1-1.pdf.

OPC. (2021). Segundo monitoreo nacional trabajadores de la cultura. Resultados, febrero 2021. Observatorio de Políticas Culturales. Recuperado de http://www.observatoriopoliticasculturales.cl/wp-content/uploads/2021/02/2do-Monitoreo-de-Trabajadores.pdf

OPC \& IMICHILE. (2016). La industria musical independiente en Chile. Cifras y datos para una caracterización. Observatorio de Políticas Culturales e Industria Musical Independiente Chile. Santiago: SCD. Recuperado de http://www.observatoriopoliticasculturales. cl/publicacion-imi-opc_versiononline_24-04/

Peters, T. (2020). Políticas culturales y desigualdad en Chile: apuntes desde un estado de emergencia. Revista Pléyade, 2020, 253-261. http://www.revistapleyade.cl/wp-content/uploads/Pleyade-especial-Revueltas-en-Chile-2019-020.pdf

Pinochet, C. (2020). Antropologías del arte en tiempo presente: una aproximación desde sus nudos problemáticos. Revista Antropologías del Sur, 7 (13), 63-78. https://doi. org/10.25074/rantros.v7i13.1729

Platea Magazine. (13 marzo, 2020). “El gobierno alemán promete ayudas a artistas y entidades musicales tras las cancelaciones por coronavirus". Recuperado de. https://www.plateamagazine.com/noticias/8546-el-gobierno-aleman-promete-ayudas-a-artistasy-entidades-musicales-tras-las-cancelaciones-por-coronavirus?fbclid=lwAR188 _ s4tt39PdklMIPU1992o-8M99_2X8xg_80yNLcnL-Q00yl8k7c580

Riethof, M. (25 octubre, 2019). Chile protests escalate as widespread dissatisfaction shakes foundations of country's economic success story. The Conversation. Recuperado de https://theconversation.com/chile-protests-escalate-as-widespread-dissatisfaction-shakes-foundations-of-countrys-economic-success-story-125628?utm _ source=twitter\&utm_medium=twitterbutton

Salazar, G. (2012). Movimientos sociales en Chile: Trayectoria histórica y proyección política. UQBAR.

SINAMUARCHI. (2020). “Comunicado a la opinión pública”. Sindicato Nacional de Músicos y Artistas de Chile. Recuperado de http://sinamuarchi.cl/web/index.php/2020/05/27/ comunicado-a-la-opinion-publica/.

Solnit. R. (2020). Un paraíso en el infierno: Las extraordinarias comunidades que surgen en el desastre. Capitan Swing.

Solo Artistas Chilenos. (19 marzo, 2020). “Artistas exigen medidas de protección al Ministerio de las Culturas, las Artes y el Patrimonio por coronavirus". Recuperado de https:// soloartistaschilenos.cl/organizaciones-artisticas-declaracion-coronavirus/.

Soto, D. (2020, 30 septiembre). Entrevistado por las autoras, Santiago de Chile.

Stahl, M. (2013). Unfree Masters: Recording Artists and the Politics of Work. Duke University Press.

TRAMUS. (2019). Red de Trabajadoras de la Música y Disidencias. Recuperado de https:// www.tramus.cl.

TRAMUS. (21 marzo, 2020a). Entre todes nos cuidamos. [Imagen adjunta]. [Actualización de estado]. Facebook. https://www.facebook.com/trabajadorasmusica/photos/pcb.14 $4489007055385 / 144488970388722$

TRAMUS. [Qtrabajadorasmusica]. (22 marzo, 2020b). TRAMUS, Trabajadoras de la música, mujeres y disidencias. [Imagen adjunta]. [Imagen]. Instagram. https://www.instagram.com/p/B-DnwZIJG9u/ 
TRAMUS. (10 abril, 2020c). Aprende junto a TRAMUS. [Imagen adjunta]. [Actualización de estado]. Facebook. https://www.facebook.com/trabajadorasmusica/photos/a.1059124 44246375/150395559798063/?type=3\&theater

Tsioulakis, I. (2020a). Musicians in Crisis. Working and Playing in the Greek Popular Music Industry. Abingdon, Oxon y New York: Routledge.

Tsioulakis, I. (16 diciembre, 2020b). "How Greek musicians weathered an economic crisis could help UK performers help COVID fall-out". The Conversation. 16 de diciembre de 2020. Recuperado de https://theconversation.com/how-greek-musicians-weathered-aneconomic-crisis-could-help-uk-performers-handle-covid-fall-out-152125/

Urqueta, E. (2019). El aporte del programa estatal Escuelas de Rock en la formación y condiciones laborales del músico popular. Una aproximación desde la política cultural chilena de posdictadura. (Tesis de maestría). Pontificia Universidad Católica de Chile. Recuperada de https://repositorio.uc.cl/handle/11534/22956

Videla, M. (17 septiembre, 2020). Entrevistada por las autoras, Santiago de Chile.

Williamson, J., \& Cloonan, M. (2016). Players' work time: A history of the British Musicians' Union, 1893-2013. Manchester University Press.

Wikström, P. (2013). La industria musical en una era de distribución digital. Clambio: 19 ensayos clave sobre cómo internet está cambiando nuestras vidas. BBVA.Recuperado de https://www.bbvaopenmind.com/articulos/la-industria-musical-en-una-era-dedistribucion-digital/

- Sobre las autoras:

Eileen Karmy es académica del Centro de Estudios Avanzados y Departamento de Artes Integradas de la Universidad de Playa Ancha. Socióloga de la Universidad Alberto Hurtado, Magíster en Artes mención Musicología de la Universidad de Chile y Doctora en Música por la Universidad de Glasgow. Investiga sobre música popular, su historia social y significaciones políticas, el trabajo y organización gremial de músicos.

Estefanía Urqueta Contreras se tituló de Pedagogía en Historia, Geografía y Ciencias Sociales de la Universidad Católica de Valparaíso. Es Magíster en Artes mención Música de la Universidad Católica de Chile. Profesora de historia, educadora artística e investigadora sobre música comunitaria, organizaciones laborales de músicos y políticas culturales.

\section{- ¿Cómo citar?}

Karmy, E. \& Urqueta, E. (2020). Música en tiempos de crisis: precariedades del trabajo artístico y resurgimiento del apoyo mutuo en Chile. Comunicación y Medios. (44), 93-105. https://doi.org/10.5354/0719-1529.2021.61381 